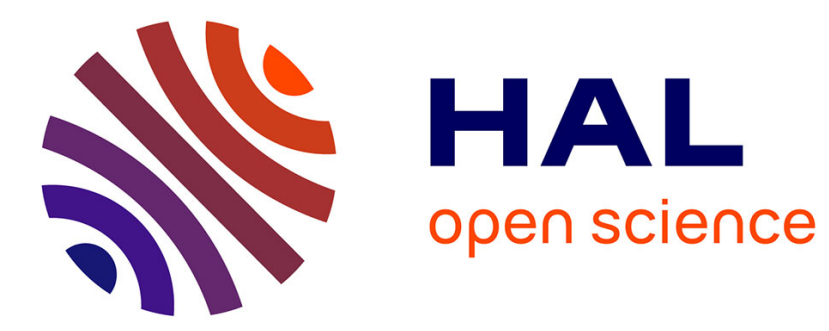

\title{
Connecting a Human Limb to an Exoskeleton
}

Nathanael Jarrasse, Guillaume Morel

\section{To cite this version:}

Nathanael Jarrasse, Guillaume Morel. Connecting a Human Limb to an Exoskeleton. IEEE Transactions on Robotics, 2012, 28 (3), pp.697-709. 10.1109/TRO.2011.2178151 . hal-02109366

\section{HAL Id: hal-02109366 https://hal.science/hal-02109366}

Submitted on 24 Apr 2019

HAL is a multi-disciplinary open access archive for the deposit and dissemination of scientific research documents, whether they are published or not. The documents may come from teaching and research institutions in France or abroad, or from public or private research centers.
L'archive ouverte pluridisciplinaire HAL, est destinée au dépôt et à la diffusion de documents scientifiques de niveau recherche, publiés ou non, émanant des établissements d'enseignement et de recherche français ou étrangers, des laboratoires publics ou privés. 


\title{
Connecting a Human Limb to an Exoskeleton
}

\author{
Nathanaël Jarrassé and Guillaume Morel \\ University Pierre et Marie Curie UPMC Univ. Paris VI, ISIR (Institut des Systèmes Intelligents et de Robotique) \\ CNRS - UMR 7222 \\ 4 place jussieu, 75005 Paris - France \\ Telephone: +33.1 .44 .27 .51 .41$ \\ Emails : jarrasse@isir.upmc.fr, guillaume.morel@isir.upmc.fr
}

\begin{abstract}
When developing robotic exoskeletons, the design of physical connections between the device and the human limb it is connected to is a crucial problem. Indeed, using an embedment at each connection point leads to uncontrollable forces at the interaction port, induced by hyperstaticity. In practice, these forces may be large because in general the human limb kinematics and the exoskeleton kinematics differ. To cope with hyperstaticity, literature suggests the addition of passive mechanisms inside the mechanism loops. However, empirical solutions proposed so far lack proper analysis and generality. In this paper, we study the general problem of connecting two similar kinematic chains through multiple passive mechanisms. We derive a constructive method that allows the determination of all the possible distributions of freed Degrees of Freedom $(D o F s)$ across the different fixation mechanisms. It also provides formal proofs of global isostaticity. Practical usefulness is illustrated through two examples with conclusive experimental results: a preliminary study made on a manikin with an arm exoskeleton controlling the movement (passive mode) and a larger campaign on ten healthy subjects performing pointing tasks with a transparent robot (active mode).
\end{abstract}

Index Terms-Wearable robotic structures, exoskeleton, fixations, kinematics, hyperstaticity, isostaticity condition, biomechanics.

\section{INTRODUCTION}

Exoskeletons are being designed by researchers for a growing number of applications, ranging from military applications [3] to rehabilitation [4], [5].

For years, research has focused mainly on technological aspects (actuators, embedment, energy...) and followed a paradigm defined in [6]: "an exoskeleton is an external structural mechanism with joints and links corresponding to those of the human body". In other words, designing the kinematics of an exoskeleton generally consists of trying to replicate human limb kinematics. This creates a number of advantages: similarity of the workspaces, singularity avoidance [7], oneto-one mapping of joint force capabilities over the workspace. However, this paradigm suffers from a major disadvantage due to the impossibility of precisely replicating human kinematics with a robot. Indeed two problems occur: morphology drastically varies between subjects and, for a given subject, the joint kinematics are very complex and cannot be imitated by conventional robot joints [8]. Actually, it is impossible to find any consensual model of human kinematics in the

This work has already been partly presented at ICRA'2010 [1] and RSS'2010 [2]. biomechanics literature due to complex geometry of bone surfaces [9]. For example, different models are used for the shoulder-scapula-clavicle group [10].

Discrepancies between the two kinematic chains thus seem unavoidable. Because of the connections between multiple loops, these mismatches generate kinematic incompatibility. The resulting hyperstaticity would lead, if the connected bodies were rigid, to the impossibility of moving and to the appearance of non-controllable internal forces. In practice, however, rigidity is not infinite and mobility can be obtained thanks to deformations. When a robotic exoskeleton and a human limb are connected, these deformations are most likely to occur at the interface between the two kinematic chains, due to the low stiffness of human skin and tissues surrounding the bones [11].

Solutions found in the literature to cope with this problem vary. In the first approach, compliance can be added in order to minimize generated forces. Pneumatic systems were thus added to introduce elasticity in the robot fixations and adaptability to variable limb section [12].

The second approach consists of designing the exoskeleton in such a way that adaptation to human limb kinematics is maximized. Two methods can then be employed: adaptation capability of the robot serial chain can be increased (by adding adjustable length segments) or redundancy can be exploited. The latter method includes adding passive or active DoF serially in the robot kinematic chain to align active joint axes to the human joint axes [13]. These solutions tend to complicate the structure and its control. Moreover their ability to solve the problem of hyperstaticity has never been proved formally. The last approach is different and involves adding passive DoF to connect the two kinematic chains one to the other. Such a principle is common in mechanism theory: passive DoF are usually added to reduce the degree of hyperstaticity. This was proposed back in the 1970s in the context of passive orthoses, [14], [15]. More recently, this principle was used for the design of a one degree of freedom active device in [11]. To the authors' knowledge, this was the first study in robotic exoskeleton design explicitly evoking the problem of hyperstaticity in force transmission and proposing to add passive DoFs. However, in [11], force transmission was analyzed only in a plane, thus neglecting the off-plane forces arising from the unavoidable lack of parallelism between the human limb plane and the exoskeleton plane. Furthermore, the study relies on explicit equations derived for a particular mechanism. 
In contrast, the constructive method proposed in this paper applies to a general spatial problem, which is fully formalized and then solved thanks to a set of necessary and sufficient conditions for global isostaticity (Section II). In Sec. III, the method is applied to the ABLE exoskeleton, a given active 4DoF arm exoskeleton. In Section IV, experimental results illustrate the practical interest of the approach.

\section{General Method}

The main question addressed in this paper is: given a proposed exoskeleton structure designed to (approximately) replicate a human limb kinematic model, how can we connect it to the human limb while avoiding the appearance of uncontrollable forces at the interface? The answer takes the form of a set of passive frictionless mechanisms used to connect the robot and the subject's limb that allows the avoidance of hyperstaticity.

\section{A. Problem formulation}

Let us consider two different serial chains with multiple couplings as illustrated in Fig. 1. One represents a human $\operatorname{limb} \mathbf{H}$ and the other the robot structure $\mathbf{R}$.

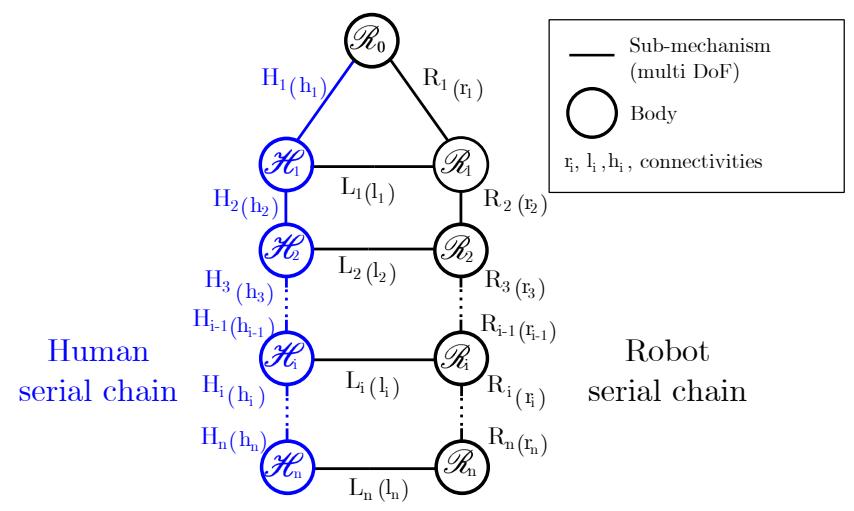

Fig. 1. Schematic of two serial chains with parallel coupling

Assuming that the base body of the exoskeleton is attached to the body of a human subject and that this common body is denoted $\mathscr{R}_{0} \equiv \mathscr{H}_{0}$, we will consider that the robot and the limbs are connected through $n$ fixations. Each fixation is a mechanism $\mathbf{L}_{i}$ for $i \in\{1, . ., n\}$ consisting in a passive kinematic chain which connects a human body $\mathscr{H}_{i}$ to a robot body $\mathscr{R}_{i}$. Mechanisms $\mathbf{L}_{i}$ are supposed to exhibit a connectivity $l_{i}$. Recall that connectivity is the minimum and necessary number of joint scalar variables that determine the geometric configuration of the $\mathbf{L}_{i}$ chain [16]. Typically, $\mathbf{L}_{i}$ will be a non-singular serial combination of $l_{i}$ one DoF joints. The fixation can be an embedment $\left(l_{i}=0\right)$ or can release several DoFs, such that:

$$
\forall i \in\{1, . ., n\}, \quad 0 \leq l_{i} \leq 5 .
$$

Indeed choosing $l_{i} \geq 6$ would correspond to complete freedom between $\mathscr{H}_{i}$ and $\mathscr{R}_{i}$ which would not make any practical sense in the considered application where force transmission is required.
Between $\mathscr{R}_{i-1}$ and $\mathscr{R}_{i}$, on the robot side, there is an active mechanism $\mathbf{R}_{i}$, the connectivity of which is denoted by $r_{i}$. Similarly, between $\mathscr{H}_{i-1}$ and $\mathscr{H}_{i}$ on the human side, there is a mechanism $\mathbf{H}_{i}$ of connectivity $h_{i}$. Note that due to the complexity of human kinematics, $h_{i}$ is not always exactly known. Literature from biomechanics provides controversial data on this point. For example, the elbow is often modeled as a one DoF joint, but in reality a residual second DoF can be observed [17].

Our goal is to design mechanisms $\mathbf{L}_{i}$ with $i \in\{1, . ., n\}$ in such a way that all the forces generated by the exoskeleton on the human limb are controllable and that there is no possible motion for the exoskeleton while the human limb is still. We shall thus consider next that the human limbs are virtually attached to the base body $\mathscr{R}_{0}$. This represents the case when the subject does not move at all. The resulting system, depicted in Fig. 2, is denoted by $S_{n}$.

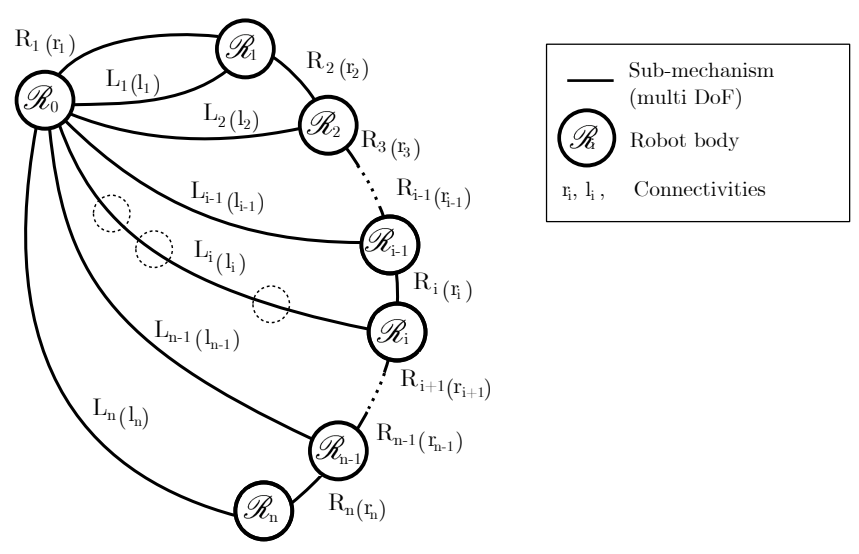

Fig. 2. Studied problem with a fixed human limb

In order to study the mobility of such a complex multi-loop mechanism, scalar mobility indexes obtained by counting the number of loops, the number of individual DoFs and the number of rigid bodies cannot be applied. Rather, mobility analysis has to be performed by exploiting a more general method from the theory of mechanisms. A number of approaches can be found in the literature, from linear transformations [18] to Lie algebra [19]. For this study, analyzing the rank of the spaces of twists and wrenches, as proposed in [20] was found to be convenient and efficient.

A proper design for the passive mechanisms $\mathbf{L}_{i}$ shall guarantee that, in the absence of any external forces, both:

$$
\begin{aligned}
& \forall i \in 1 \cdots n, \quad{ }^{\mathbf{S}_{n}} T_{i}=\{0\} \quad \text { and } \\
& \forall i \in 1 \cdots n, \quad{ }^{\mathbf{S}_{n}} W_{\mathbf{L}_{i} \rightarrow 0}=\{0\},
\end{aligned}
$$

where ${ }^{\mathbf{S}_{n}} T_{i}$ is the space of twists describing the velocities of robot body $\mathscr{R}_{i}$ relative to $\mathscr{R}_{0}$ when the whole mechanism $\mathbf{S}_{n}$ is considered and ${ }^{\mathbf{S}_{n}} W_{\mathbf{L}_{i} \rightarrow 0}$ is the space of wrenches (forces and moments) statically admissible transmitted through the $\mathbf{L}_{i}$ chain on the reference body $\mathscr{R}_{0}$, when the whole mechanism $\mathbf{S}_{n}$ is considered.

Equation (2a) expresses the fact that the mobility of any robot body connected to a human limb should be null, which is a required condition since we are assuming that the human 
member is still. Moreover, equation (2b) imposes that, considering the whole mechanism, there can be no forces of any kind exerted on the human limb. Indeed, since the actuators are applying null generalized forces, any force at the connection ports would be uncontrollable due to hyperstaticity. In the next Equation (2) is referred to as the global isostaticity condition.

\section{B. Conditions on the twists space ranks}

At first, one can notice the recursive structure of the considered system: let $\mathbf{S}_{i}$ be the sub-mechanism constituted by the bodies $\mathscr{R}_{0}$ to $\mathscr{R}_{i}$, the chains $\mathbf{R}_{0}$ to $\mathbf{R}_{i}$ and $\mathbf{L}_{0}$ to $\mathbf{L}_{i}$. The system $\mathbf{S}_{i}$ can be represented recursively from $\mathbf{S}_{i-1}$, as in Fig. 3, where $m_{i-1}$ is the connectivity of $\mathbf{S}_{i-1}$. In this convention, $\mathbf{S}_{0}$ represents a zero DoF mechanism. Using this recursive

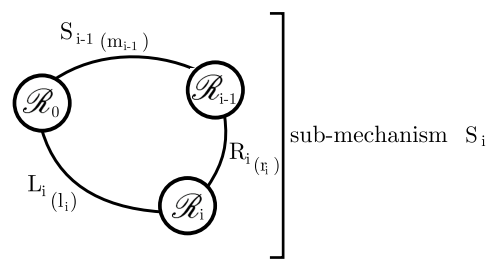

Fig. 3. Recursive structure $\mathbf{S}_{i}$ of the system

representation one can establish the following proposition:

Proposition 1: The conditions (2) are equivalent to :

$$
\begin{array}{cc}
\forall i \in 1 \cdots n, \quad \operatorname{dim}\left(T_{\mathbf{S}_{i-1}}+T_{\mathbf{R}_{i}}+T_{\mathbf{L}_{i}}\right)=6 & \text { and } \\
\forall i \in 1 \cdots n, \quad \operatorname{dim}\left(T_{\mathbf{S}_{i-1}} \cap T_{\mathbf{R}_{i}}\right)=0 & \text { and } \\
\operatorname{dim}\left(T_{\mathbf{S}_{n}}\right)=0, &
\end{array}
$$

where $T_{\mathbf{S}_{j}}=\mathbf{S}_{j} T_{j}$ is the space of twists describing the velocities of $\mathscr{R}_{j}$ relative to $\mathscr{R}_{0}$, when $\mathbf{S}_{j}$ is considered isolated from the rest of the mechanism (then it is different from ${ }^{S_{n}} T_{j}$ ), $T_{\mathbf{R}_{i}}$ is the space of twists produced by $\mathbf{R}_{i}-i$.e. the space of twists of $\mathscr{R}_{i}$ relative to $\mathscr{R}_{i-1}$ if they were only connected through $\mathbf{R}_{i}, T_{\mathbf{L}_{i}}$ is the space of twists produced by $\mathbf{L}_{i}$ i.e. the space of twists of $\mathscr{R}_{i}$ relative to $\mathscr{R}_{0}$ if they were only connected through $\mathbf{L}_{i}$.

The demonstration can be found in Appendix A.

Physical interpretation can be obtained by observing Fig. 3. Equation (3a) imposes that the mobility for the open chain $\mathbf{S}_{i-1}-\mathbf{R}_{i}-\mathbf{L}_{i}$ is 6 . Otherwise, the closed loop sub-mechanism $\mathbf{S}_{i}$ represented in Fig. 3 would be hyperstatic. This condition will impose a minimal mobility to be recursively added to the system. Equation ( $3 b$ ) imposes that, when the body $\mathscr{R}_{i}$ is still, there is no possible motion for $\mathscr{R}_{i-1}$. Otherwise, the system would exhibit too much mobility, i.e. an uncontrolled motion would be observed for at least body $\mathscr{R}_{i-1}$ in the global system. This condition will impose a maximal mobility to be recursively added to the system. Finally, Equation (3c) imposes that the last robot body cannot move, which is trivial.

Remarkably, conditions (3) involve the space of twists generated by $\mathbf{R}_{i}$ and $\mathbf{L}_{i}$ when taken isolated, which is of great help for design purposes. In the next subsection, we convert these conditions into constraints on the connectivities $r_{i}=\operatorname{dim}\left(T_{\mathbf{R}_{i}}\right)$ and $l_{i}=\operatorname{dim}\left(T_{\mathbf{L}_{i}}\right)$. To do so, we suppose that kinematic singularities are avoided. In other words, summing the subspaces of twists will always lead to a subspace of maximum dimension given the dimensions of individual subspaces. This hypothesis will lead to determine the number of DoFs that will be included in the passive fixation mechanisms $\mathbf{L}_{i}$. Of course as it is usual in mechanism design, when a particular design is finally proposed, it will be necessary to verify a posteriori the singularity avoidance condition.

\section{Conditions on connectivity}

At first, let us compute the connectivity of $\mathbf{S}_{i}$. One has:

$$
T_{\mathbf{S}_{i}}=T_{\mathbf{L}_{i}} \cap\left(T_{\mathbf{R}_{i}}+T_{\mathbf{S}_{i-1}}\right),
$$

which directly results from the space sum law for serial chains and the intersection law for parallel chains (see [20]). Furthermore, since for any vector subspaces $\mathbf{A}$ and $\mathbf{B}, \operatorname{dim}(\mathbf{A})+$ $\operatorname{dim}(\mathbf{B})=\operatorname{dim}(\mathbf{A}+\mathbf{B})+\operatorname{dim}(\mathbf{A} \cap \mathbf{B})$, one gets:

$$
\begin{aligned}
m_{i}= & \operatorname{dim}\left(T_{\mathbf{S}_{i}}\right) \\
= & \operatorname{dim}\left(T_{\mathbf{L}_{i}}\right)+\operatorname{dim}\left(T_{\mathbf{R}_{i}}+T_{\mathbf{S}_{i-1}}\right)-\operatorname{dim}\left(T_{\mathbf{L}_{i}}+T_{\mathbf{R}_{i}}+T_{\mathbf{S}_{i-1}}\right) \\
= & \operatorname{dim}\left(T_{\mathbf{L}_{i}}\right)+\operatorname{dim}\left(T_{\mathbf{R}_{i}}\right)+\operatorname{dim}\left(T_{\mathbf{S}_{i-1}}\right)-\operatorname{dim}\left(T_{\mathbf{R}_{i}} \cap T_{\mathbf{S}_{i-1}}\right) \\
& -\operatorname{dim}\left(T_{\mathbf{L}_{i}}+T_{\mathbf{R}_{i}}+T_{\mathbf{S}_{i-1}}\right) .
\end{aligned}
$$

If condition (3) is respected then $\operatorname{dim}\left(T_{\mathbf{R}_{i}} \cap T_{\mathbf{S}_{i-1}}\right)=0$ and $\operatorname{dim}\left(T_{\mathbf{L}_{i}}+T_{\mathbf{R}_{i}}+T_{\mathbf{S}_{i-1}}\right)=6$. Therefore, under full rank assumption, one gets:

$$
m_{i}=l_{i}+r_{i}+m_{i-1}-6
$$

Finally, using $m_{0}=0$, this recursive equation simplifies to:

$$
m_{i}=\sum_{j=1}^{i}\left(l_{j}+r_{j}\right)-6 . i
$$

Now that an expression for $m_{i}$ has been obtained, it is possible to convert Eq. (3) into conditions on $l_{i}$ and $r_{i}$. First, from Equation (3a), noticing that any vector subspaces $\mathbf{A}, \mathbf{B}$ and $\mathbf{C}$ of a vector space $\mathbf{E}, \operatorname{dim}(\mathbf{A}+\mathbf{B}+\mathbf{C}) \leq \operatorname{dim}(\mathbf{A})+\operatorname{dim}(\mathbf{B})+$ $\operatorname{dim}(\mathbf{C})$, it is necessary that:

$$
\forall i \in 1 \cdots n, \quad m_{i-1}+r_{i}+l_{i} \geq 6, \quad \text { or }: \quad \sum_{j=1}^{i}\left(l_{j}+r_{j}\right) \geq 6 . i
$$

Moreover, if $\mathbf{A}$ and $\mathbf{B}$ are two vector subspaces of $\mathbf{E}$ and $\operatorname{dim}(\mathbf{A})+\operatorname{dim}(\mathbf{B})>\operatorname{dim}(\mathbf{E})$, then $\mathbf{A} \cap \mathbf{B} \neq\{0\}$, Equation (3b) imposes that:

$$
\forall i \in 1 \cdots n, \quad m_{i-1}+r_{i} \leq 6 \text { or }: \sum_{j=1}^{i-1}\left(l_{j}+r_{j}\right)+r_{i} \leq 6 . i
$$

Finally, thanks to the recursive application of mobility equation to each partial chain, the last condition (3c) leads to:

$$
m_{n}=0 \text { or }: \sum_{j=1}^{n}\left(l_{j}+r_{j}\right)=6 . n
$$

Notice that (9) provides the total number of DoFs to be freed for the mechanism $\mathbf{S}_{n}$, while (7) gives the minimal value (to prevent from hyperstaticity in the sub-mechanisms $\mathbf{S}_{j}$ ) for $l_{j}$ and (8) provides the maximal one (to prevent from internal mobility in $\mathbf{S}_{j}$ ).

Thanks to these three last necessary conditions, we are able 
to calculate the different possible solutions for distributing the additional passive DoFs over the structure:

- the possible choices for $l_{1}$ are such that $5 \geq l_{1} \geq 6-r_{1}$.

- for each choice of $l_{1}$, the possible choices for $l_{2}$ are such that $5 \geq l_{2} \geq 12-r_{1}-r_{2}-l_{1}$.

This leads to a tree that groups all the admissible combinations for $l_{i}$, as illustrated in Fig. (4).

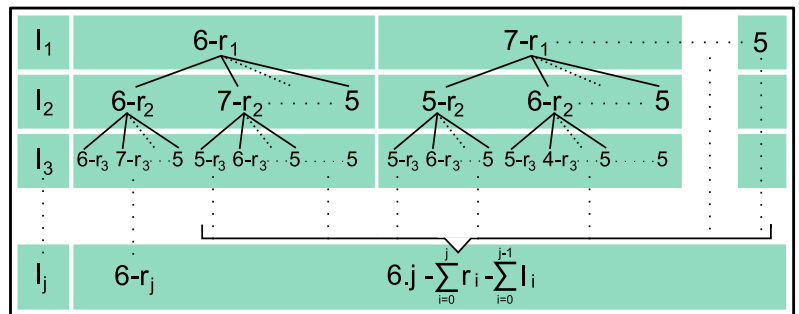

Fig. 4. Tree of possible solutions for the number of passive DoFs to add at every fixation point

Out of this tree, all the possible combinations of connectivity for the fixations are given. Of course, the selection among these solutions is to be made depending on the exoskeleton kinematics.

\section{Choosing appropriate passive DoF for the fixations}

Considering human kinematics and the three aspects of interaction (kinematic, static and physiological) simply allows us to choose the distribution and the nature of the passive DoF fixations.

Firstly, from the kinematic point of view, the rank analysis should help in the choice of the DoF to be freed. It is generally easy to determine the DoF that will increase the kinematic rank of the system and the ones that will not impact it. Velocities of the considered human limbs that are not compatible with the robot kinematics (or that can not be controlled by it) has to be allowed, and thus the fixation DoF compatible with these velocities should be freed.

Secondly, considering the force transmission, the knowledge of the forces that have to be controlled by the robot actuators allows the determination of the fixation DoF that should not be freed in order to keep the control on the human limb.

Finally, human physiology imposes constraints, especially human tissues. The human member's segments can generally be approximated by solids of revolution. To transmit forces on such segments, fixations must therefore surround the member. These fixations convert forces and moments generated by the robot into pressures applied through the surface of splints. Specific considerations have to be taken into account in order to preserve human tissues from high pressures. Considering the limb segment as a solid of revolution with axis $\Delta$, four kinds of stresses can be applied by the robot: forces perpendicular to $\Delta$, forces along $\Delta$, moments around the axis perpendicular to $\Delta$ and moment around $\Delta$.

- Forces perpendicular to $\Delta$ can be applied, but interaction surfaces need to be large on the human body in order to minimize the contact pressure level. Nevertheless, these surfaces should not be too large, so as not to completely cover the whole limb and especially some muscular areas where important volume variation occurs during

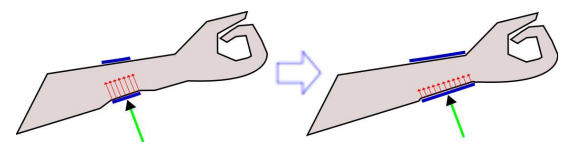

Fig. 5. Tissue deformation and the feeling of applied pressure can be high with small contact surfaces badly positioned

movement. In order to maximize the force transmission from the robot to the human, fixations should be also positioned on high stiffness areas with low sensitivity tissue. Several studies have been done on localizing these specific human body areas. For example, on the arm, the wrist is a good place to fix a splint and limit discomfort [7].

- Forces applied along $\Delta$ must be avoided. The human body structure is made of ball-joints and segments, and so the translations along limb main axis directions are not among the possible movements to be assisted. If this DoF is not released, hyperstaticity will directly generate force along this axis when the serial chains will move (See Fig. 6). Moreover, directly applying these kinds of forces through a tight fixation leads to a transmission by

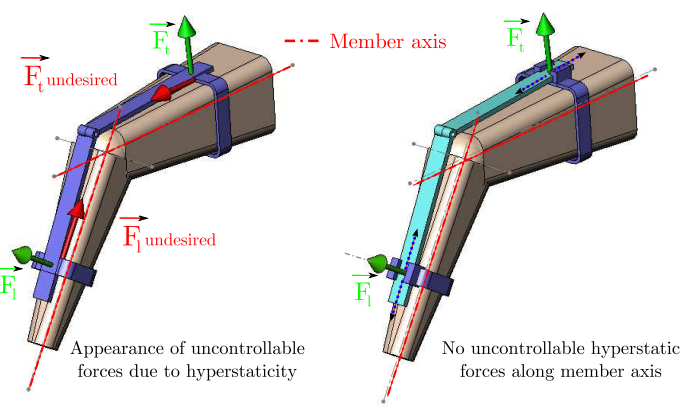

Fig. 6. Release of translations along limb segment main axis prevent hyperstatic force from occuring

friction that can generate high tangential forces on the skin, and thus, pain or at least discomfort.

- Moments around an axis perpendicular to $\Delta$ should be carefully applied: as illustrated in Fig. 7, applying such a moment results in the concentration of the stress applied to the limb tissues at two opposite points. The local forces may be rather high since the dimensions of the parts in contact with the limb shall remain small for ergonomic purposes and to keep constant contact stiffness. Moreover,

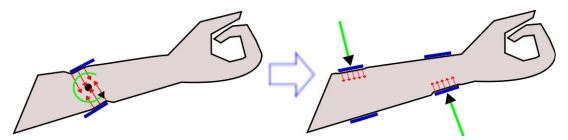

Fig. 7. Using a couple of forces instead of moments to limit stress concentration

it is often possible to use a couple of forces applied to two 
segments in order to create a torque around a limb axis. In terms of local deformations of the skin and muscles, it is highly preferable.

- Moments around limb main axis should not be transmitted. Indeed, transmitting a torsion around segment main axis would generate large deformations of the muscles, thus involving a large fiber elongation (see Fig. 8). Also,

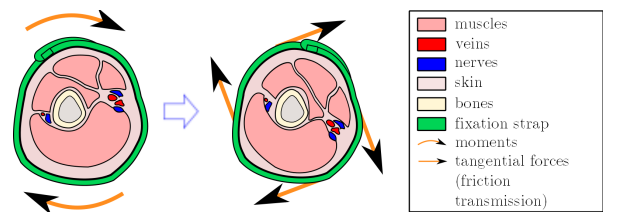

Fig. 8. Transmitting moments around the limb axis involves large tissue deformations

once again, applying this moment directly through a tight fixation is a transmission by friction.

In the next section, all these rules are applied, for the sake of illustration in a particular example.

\section{Application to A GIVEN EXOSKELETON}

\section{A. ABLE: an upper limb exoskeleton for rehabilitation}

ABLE (see Fig. 9) is a 4-axis exoskeleton that has been designed by CEA-LIST on the basis of an innovative screwand-cable actuation technology [21]. Its kinematics are composed of a shoulder spherical joint comprising 3 coincident actuated pivots and a 1 DoF actuated pivot elbow. The forearm,

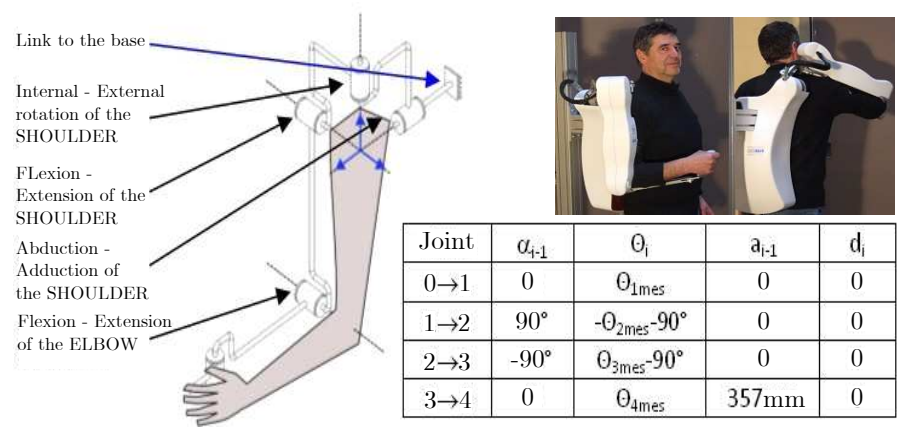

Fig. 9. Kinematics of ABLE

terminated by a handle, is not actuated. Details on this robot can be found in [22].

\section{B. Fixation design of $A B L E$}

In this section, we apply the general method proposed in Sec. II to ABLE. We proceed in three steps:

- build the tree of possible values for $l_{i}$

- choose a preferred solution among them by examining force transmission properties and kinematic complementarity

- verify the full kinematic rank which is reported in Appendix B.

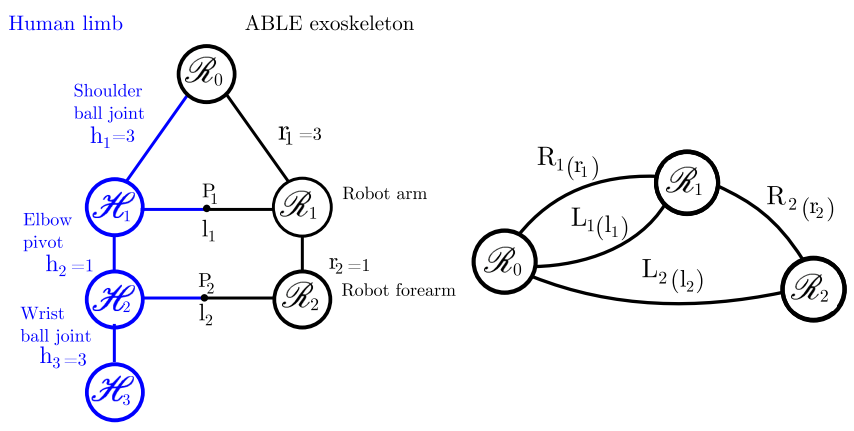

Fig. 10. Schematic of ABLE and human arm coupling

Firstly, since ABLE comprises an upper arm and a forearm, we shall use two fixations (See Fig 10). The total number of passive DoF to be added is given by Equation (9):

$$
\sum_{j=1}^{n=2} l_{j}=12-\sum_{j=1}^{n=2} r_{j}=12-(3+1) \quad \Rightarrow l_{1}+l_{2}=8
$$

Moreover, for the first fixation, Equation (7) and (8) give:

$$
6-r_{1} \leq l_{1} \leq 5 \quad \Rightarrow \quad 3 \leq l_{1} \leq 5 .
$$

Since the total number of DoFs is fixed, the tree of possible solutions consists here of three parallel branches where $l_{1}$ is chosen between 3 and 5 and $l_{2}=8-l_{1}$. Possible couples for $\left(l_{1}, l_{2}\right)$ are $(3,5),(4,4)$ and $(5,3)$. Hereafter, these three options are analyzed in order to choose a preferred design from among them.

- Case a: $l_{1}=3$ and $l_{2}=5$. In this case, both $\mathbf{S}_{1}$ taken alone and $\mathbf{S}_{2}$ are isostatic, which corresponds to the most intuitive way of achieving global isostaticity. Degrees of Freedom for $\mathbf{L}_{1}$ must be chosen complementary to those of $\mathbf{R}_{1}$ in order to satisfy the full rank assumption. Since $\mathbf{R}_{1}$ is a ball joint that generates three independent rotational velocities around its center $M_{1}, \mathbf{L}_{1}$ must generate three independent velocities at point $M_{1}$. For example, three non coplanar translations could be used for $\mathbf{L}_{1}$. However, in this case, the fixation would transmit a null force, i.e. a pure couple. This seems undesirable due to the torsion of the soft tissues that it would create around $P_{1}$ at the level of the attachment to the limb. One could thus think of using, for $\mathbf{L}_{1}$, a ball joint around $P_{1}$, but in this case, the full rank condition would not be respected, because $\mathbf{R}_{1}$ and $\mathbf{L}_{1}$ would both generate the same rotation around $\vec{z}_{1}=\frac{1}{\left\|\overrightarrow{M_{1} P_{1}}\right\|} \overrightarrow{M_{1} P_{1}}$. Finally, the preferred solution is to choose for $\mathbf{L}_{1}$ two pivot joints perpendicular to the arm main axis $\vec{z}_{\text {arm }}$, and one translation joint collinear $\vec{z}_{\text {arm }}$. In this case, two forces perpendicular to $\vec{z}_{\text {arm }}$ and one moment around $\vec{z}_{\text {arm }}$ can be exchanged between the exoskeleton and the arm through $\mathbf{L}_{1}$. Moreover, since $\mathbf{S}_{1}$ is isostatic, one has $m_{1}=0$. Therefore $\mathbf{L}_{2}$ needs to be designed in order to be kinematically complementary to $\mathbf{R}_{2}$, which is a pivot of axis $\left(M_{2}, \vec{z}_{e}\right)$. A simple solution is to choose a ball joint around $P_{2}$, and two sliders whose support vector generate a plane that is perpendicular to the velocity generated at $P_{2}$ by the elbow pivot joint at $\left(M_{2}, \vec{z}_{e}\right)$. The resulting overall design is noted (a) and represented in Figure 11.

- Case b: $l_{1}=4$ and $l_{2}=4$. Note that in this case, $\mathbf{S}_{1}$ taken 

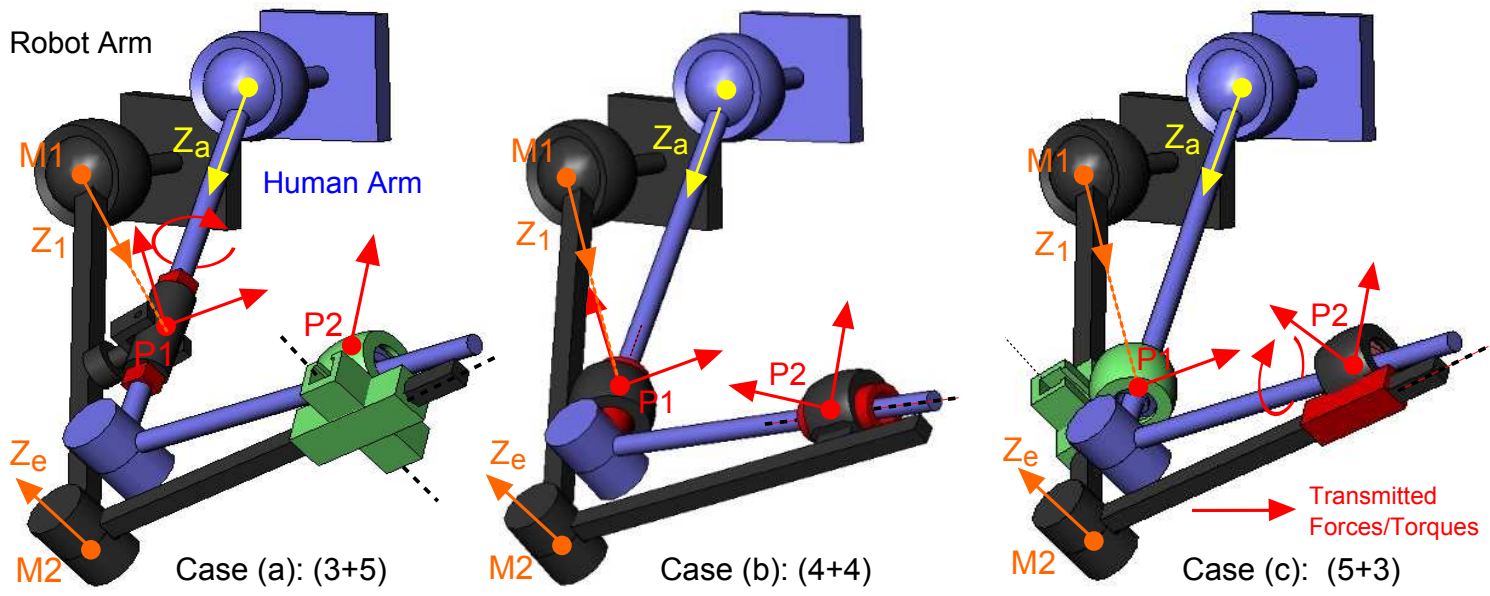

Fig. 11. Three options for coupling ABLE to a human arm. Case (a): the 3 DoF upper arm fixation mechanism combines one universal joint and one slider while the 5 DoF lower arm fixation mechanism includes one ball joint and two sliders; case (b): both the 4 DoF fixation mechanisms combine one ball joint and one slider; case (c): symmetrically to case (a), the 5 DoF upper arm fixation mechanism combines one ball joint and two sliders while the 3 DoF lower arm fixation mechanism includes one universal joint and one slider. Arrows in red represent the forces and moments that can be transmitted through the passive fixations, which are complementary to the passive DoF.

alone is a 1 DoF mechanism, while only $\mathbf{S}_{2}$ is isostatic. We consider solution (a), for which one DoF must be added to $\mathbf{L}_{1}$ and one must be removed from $\mathbf{L}_{2}$. Concerning $\mathbf{L}_{1}$, keeping freed the 3 DoF liberated for the isostatic solution (a), it seems preferable to choose the rotation around $z_{1}$ for the extra freed DoF. Indeed, this will cancel the local tissue torsion due to moment transmission around $\vec{z}_{1}$. As a result, $\mathbf{S}_{1}$ is now a 1 DoF mechanism consisting of a pivot around $\left(M_{1}, \vec{z}_{1}\right)$. Concerning $\mathbf{L}_{2}$, the DoF to be removed from the solution (a) will not degrade the dimension of $T_{\mathbf{S}_{1}}+T_{\mathbf{R}_{2}}+T_{\mathbf{L}_{2}}$. It seems preferable to keep the freed three rotations around $P_{2}$ and only one translation along the forearm axis $\vec{z}_{f}$. Indeed, again, this choice avoids any torsion around $P_{2}$. Furthermore, it is shown in Appendix B that singular configurations of this solution, noted (b) and represented in Figure 11 are easily identifiable and far away from nominal conditions of operation.

- Case c: $l_{1}=5$ and $l_{2}=3$. Similarly to solution (a), this combination will necessarily lead to transmit at least one torsion moment around $\vec{z}_{f}$, as illustrated in Figure 11 (solution (c)).

Finally the preferred solution is (b) because it does not involve the application of any torsion.

Note that with solution (b), generating a moment to the human upper arm around $\vec{z}_{\text {arm }}$ is obtained by applying opposite pure forces perpendicular to $\vec{z}_{\text {arm }}$ at $P_{1}$ and to $\vec{z}_{f}$ at $P_{2}$ (see Fig. 12).

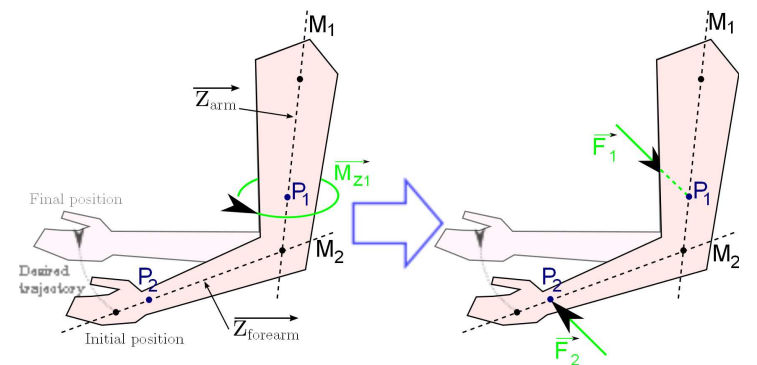

Fig. 12. Transmitting a moment around the upper arm axis with solution (b) (left) and (c) (right)
Interestingly, this reproduces the method used by physical therapists to assist patients in generating internal rotations of the shoulder without torsion to the tissue. As a price, the full extension configuration, when $M_{1}, P_{1}$ and $P_{2}$ are aligned, is singular, as detailed in Appendix $\mathrm{B}$. This configuration corresponds to the human limb singular configuration and can be easily avoided by limiting the range of the elbow extension a few degree before full extension.

\section{Fixation realization}

The two fixation mechanisms are identical. They will generate three independent rotations and one translation along the limb. The mechanism used to create this function consists of

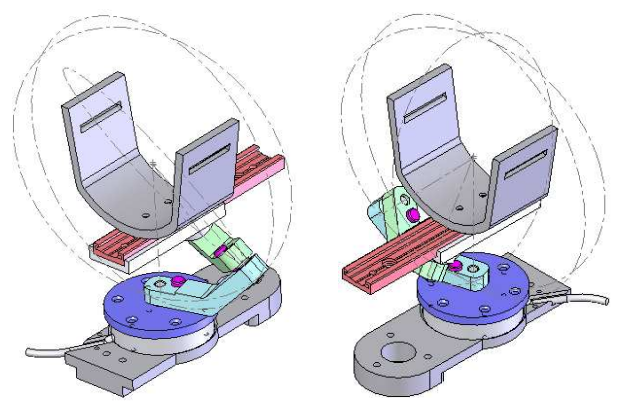

Fig. 13. Fixation simplification and realization (rear and front)

three successive pivot joints the axes of which coincide and one slider whose axis is parallel to the human limb (see Fig 13).

The fixations were dimensioned differently: one to allow forearm pronosupination and the other not to collide with arm tissues. As a result, possible motions left by the passive fixations have the ranges as shown in Table 1.

These fixations were both fitted with one force sensor placed on the base (ATI Nano43 6-axis Force/Torque sensor), allowing us to reconstruct the three force and torque components at $P_{1}$ and $P_{2}$ respectively). 


\begin{tabular}{|l|c|}
\hline DoF & Fixation \\
\hline Rotation1 $(\perp$ to the limb axis) & $360^{\circ}$ \\
\hline Rotation2 $(\perp$ to the limb axis) & $90^{\circ}$ \\
\hline Rotation3 (around the limb axis) & $110^{\circ}$ \\
\hline Translation & $100 \mathrm{~mm}$ \\
\hline
\end{tabular}

TABLE I

RANGE OF THE PASSIVE DOF FIXATION

For the experiments presented in the next section, in order to compare the forces involved with and without DoF liberation, the fixations were also equipped with removable metallic pins, allowing us to quickly lock the passive DoF without detaching the subject from the exoskeleton. These fixations were
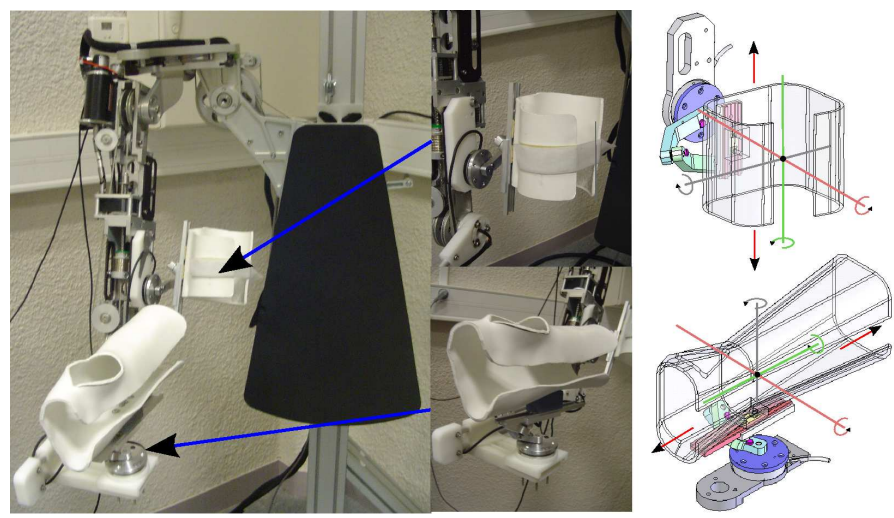

Fig. 14. The two fixations on the exoskeleton

mounted on the 4-DoF ABLE exoskeleton. Arm fixation is placed near the elbow, just under the triceps. Forearm fixation is placed near the wrist. Thermoformable materials were also used to create two splints adapted to human morphology. These splints are connected to the last fixation body. The wrist splint was specifically created to lock the wrist flexions, which are not studied here. Only passive pronosupination is allowed.

\section{EXPERIMENTAL RESULTS}

\section{A. Preliminary evaluation on manikin (passive mode)}

1) Experimental setup: An articulated manikin was used for the experiment. Its arms possess 5 passive DoF (3 rotations at the shoulder, a pivot elbow and pronosupination that was not used during experiments) and is thus adapted to our 4 DoF exoskeleton. However, several discrepancies can be observed between the robot kinematics and those of the manikin. Firstly, the manikin's elbow is not a perfect ball joint as the three axes of rotation do not exactly coincide at one point. Secondly, the manikin's elbow also suffers from backlash. Most importantly, the manikin's forearm length (approx. $290 \mathrm{~mm}$ ) is significantly shorter than the distance between the shoulder's center and the elbow pivot point on the robot side $(357 \mathrm{~mm})$. Therefore, as illustrated in Fig. 15, the distance between the robot shoulder's center (red spot) and the manikin shoulder center (green spot) reaches a few centimeters. Moreover, a large misalignment between the two elbow axes (dashed lines) can be observed in the picture on the right, whereas the axes approximately matched when the manikin was initially installed on the robot

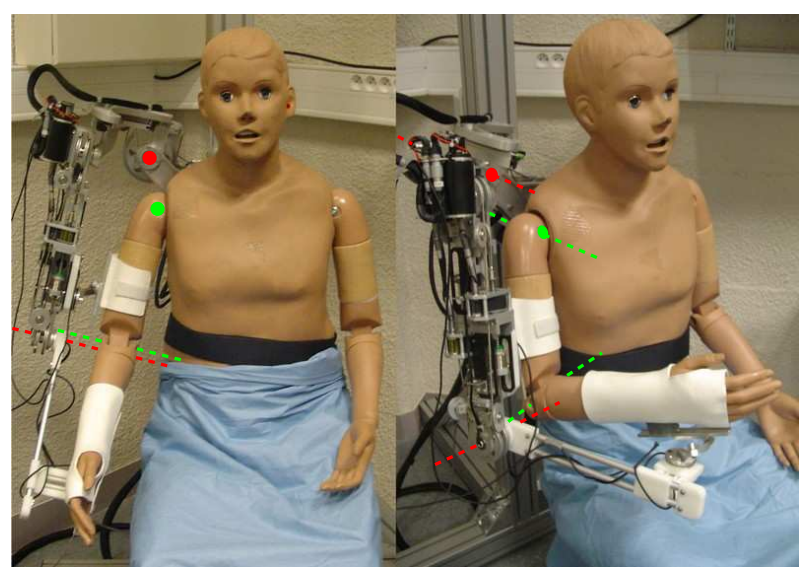

Fig. 15. A manikin connected to ABLE: the shoulder centers and the elbow axes are significantly mismatched

(left picture).

Analyzing the interaction force and torque variations at the interfaces during the same movement with the fixation mechanisms freed or locked will not only allow us to evaluate the impact on preventing the appearance of uncontrolled forces, but also to quantify them roughly.

The manikin was thus placed in the exoskeleton and attached with the two fixations. The thermoformable splints allow the avoidance of any looseness in the fixation and increase the contact stiffness (no foam needed).

During the experiments, the exoskeleton imposes a controlled trajectory, with a constant speed, to the manikin arm. The experiment consists of six simple movements that all end in the same 3D point for the end effector, but with a different arm posture (recall that the exoskeleton possesses 4 joints and therefore is redundant for a $3 \mathrm{D}$ point reaching task). The target was reached at a constant and low speed $(0.05 \mathrm{~m} / \mathrm{s})$ in order to limit inertial forces. Due to the rigidity of the manikin surface, the movement amplitude on every exoskeleton joint was limited to $15^{\circ}$ in order to limit the forces that appear during experiments. Indeed, when the exoskeleton is connected to a human limb, thanks to skin and muscle deformations, the hyperstatic force level applied on the human kinematic structure (the bones) is reduced, but with this plastic manikin, larges forces can appear.

The use of a manikin controlled by an exoskeleton allows a perfect repeatability during the experiments. This is representative of co-manipulation cases where the robot generates a controlled motion during robotic rehabilitation or movement assistance for impaired people.

2) Results and discussions: Principal results are presented below. In Fig. 16, we plotted the incompatible force absolute value (along $\overrightarrow{z_{a r m}}$ and $\overrightarrow{z_{f}}$ ) and mean moment averaged norm during the experiments for the two sensors, averaged across the six movements (moments are computed at the rotation center of the fixation). We can observe on the arm fixation a decrease in the incompatible force $\left(F_{x}\right)$ and torques by approximatively $95 \%$. For the forearm fixation, approximatively $96 \%$ decrease can be observed for the incompatible force and moment components. Figure 17 presents the norm 


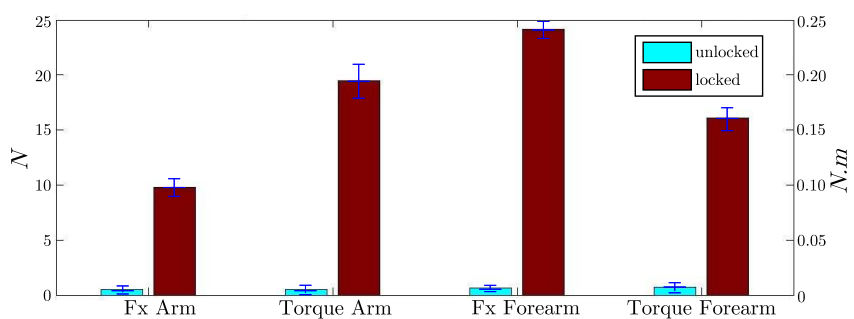

Fig. 16. Averaged absolute value of the incompatible force $\left|F_{x}\right|$ and moments norm $\sqrt{\left(M_{x}^{2}+M_{y}^{2}+M_{z}^{2}\right)}$ on the two fixations (mean for the six movements)

of the components ( $F_{y}$ and $F_{z}$, perpendicular to the human limb axis) corresponding to the components allowed to be transmitted by the passive fixations. An important decrease (up to $30 \%$ ) of the level of the forces that the exoskeleton is allowed to apply on the arm is observed with the passive DOF fixations. However it still remains small compared to the ones observed with the hyperstatic forces. Note that the decrease

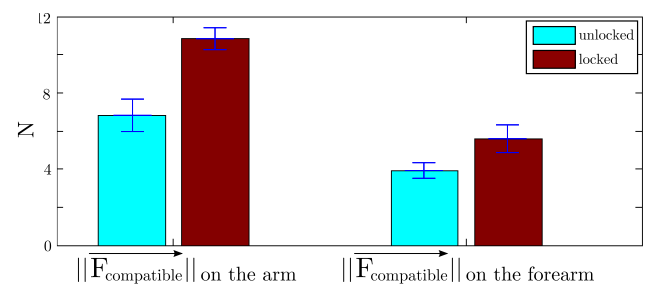

Fig. 17. Allowed forces $\left(\sqrt{\left(F_{x}^{2}+F_{y}^{2}\right)}\right)$ norm on the two fixation (mean for the six movements)

in the level of hyperstatic force achieved by the fixations resulting from our method and the obtained numerical value of the hyperstatic forces have to be interpreted. Indeed, due to the manikin arm smallness (see Fig. 15) and its body surface rigidity, hyperstatic force level is higher than it is during a comanipulation between the exoskeleton and a human subject. It is also important to notice that, even with the passive DoF fixation, residual forces remain at the two fixation points about $2 \mathrm{~N}$ of force and $0.02 \mathrm{~N} \cdot \mathrm{m}$ of torque. This can be explained by the residual friction in the fixation mechanism (which small mechanical parts, especially the bearings, are exposed to important loads) and by the fixation weight (approximately $150 \mathrm{~g}$ ) that directly applies on the sensor according to arm posture.

\section{B. Evaluation on healthy subjects (active mode)}

Since the evaluation of the fixations during a passive mode interaction has illustrated their ability to minimize the uncontrolled force level, an alternative experiment has been conducted with healthy subjects based on a generic method dedicated to the quantification of alterations in human upper limb movement during co-manipulation with exoskeletons. The method was previously presented in [23].

We propose a comparison of two performance indices detailed in the method, calculated from records of forces and movements obtained during simple pointing tasks performed by healthy subjects attached to a "transparent" exoskeleton through fixations with and without the passive DoF freed.
1) Transparency (active mode): It is essential to make the exoskeleton as transparent as possible, in order to limit the residual force level, which may appear due to gravity, inertia and friction. Here, transparency is understood as the capacity, for the robot, to not apply any resistive forces in reaction to intentional movements of the subject. Compensations were thus deployed on the robot. As ABLE is only fitted with optical encoders, we do not have access to an acceleration signal. Transparency is thus achieved by an experimentally identified gravity compensation for all axes and also by compensating for the residual dynamic dry friction compensation. This residual friction compensation has been developed in order to blend the friction phenomena on all axes, and so as not to lead subject to make non-natural movements because of joint discomfort.

2) Task and subjects: During all the experiments, we assume the exoskeleton to be "transparent" due to the gravity and friction compensation. Ten voluntary subjects were involved in this experiment. In order to exploit the robot's DoF, pointing movements were made towards four targets positioned in different parts of the workspace, allowing us to analyze the interactions between the subject and the robot when different axes of motion were involved. Three lines were drawn on the ground from the starting position, one in the para-sagittal plane and the others at $45^{\circ}$ both sides of the first line. The targets were marked on poles which were placed $50 \mathrm{~cm}$ from the starting position on each of the three lines. The target height was positioned at the level of the exoskeleton elbow axis for targets 1-3 and target 4 was positioned above target 2 , the height was equal to the horizontal distance between targets 1-2 and 2-3 (see Fig 18 and 19).

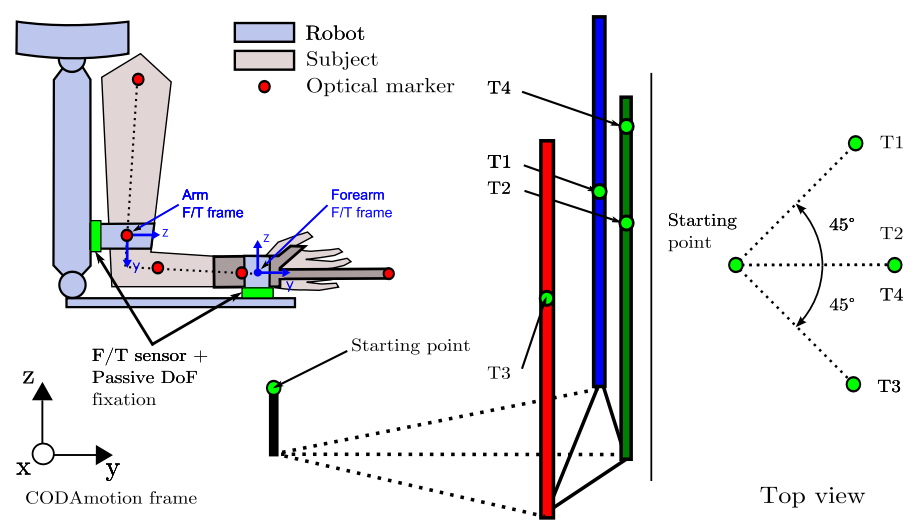

Fig. 18. Schematic of the experimental setup

The starting point was standardized with the elbow in maximum extension, the humerus vertical and the forearm in mid prone position. The subjects rested their backs against the support of the robot; a large belt was used to prevent trunk movement and a splint was used to prevent wrist motion, both of which would confound analysis of shoulder and elbow angles. A pointer was fixed to the splint.

Ten healthy volunteers aged between 22 and 30, unaware of what was being studied, were included ( 9 male and 1 female). No particular care was taken to recruit subjects with a specific morphology adapted to the exoskeleton structure. They gave informed consent according to ethical procedures. 
Subjects were allowed to practice moving with the robot for 5 minutes prior to recording. Five movements were recorded to each target. Subjects were instructed to move as naturally as possible to touch the target. A few minutes of free training

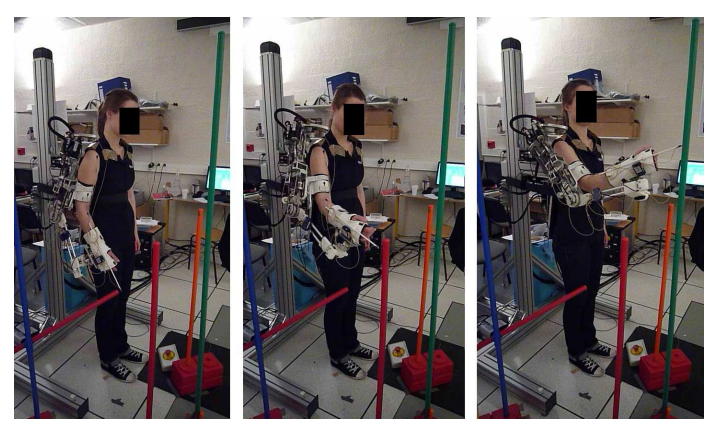

Fig. 19. A subject pointing to different targets wearing the exoskeleton

allow the subjects to feel comfortable and safe with the device since initial movements may be perturbed by the newness of the experience. A good indicator that the subject is ready to perform the experiment is when he or she feels safe and when the movements between two targets are qualitatively repeatable.

3) Results: We first present the results obtained across the 40 trials for one single representative subject. Figure 20 shows the average amount of force and moment appearing along the directions where they are not controllable, for one subject, during every trial to each target ( 5 trials to 4 different targets under 2 conditions). The general tendency is that the amount of force or moment is larger in the red bars (fixations locked) than in blue bars (fixations freed). Also, for a given condition and a given target, only small variations can be observed between the 5 bars. This tends to show that the decrease of the force level does not result from a learning phenomenon. Rather, it is effectively due to the passive fixations.
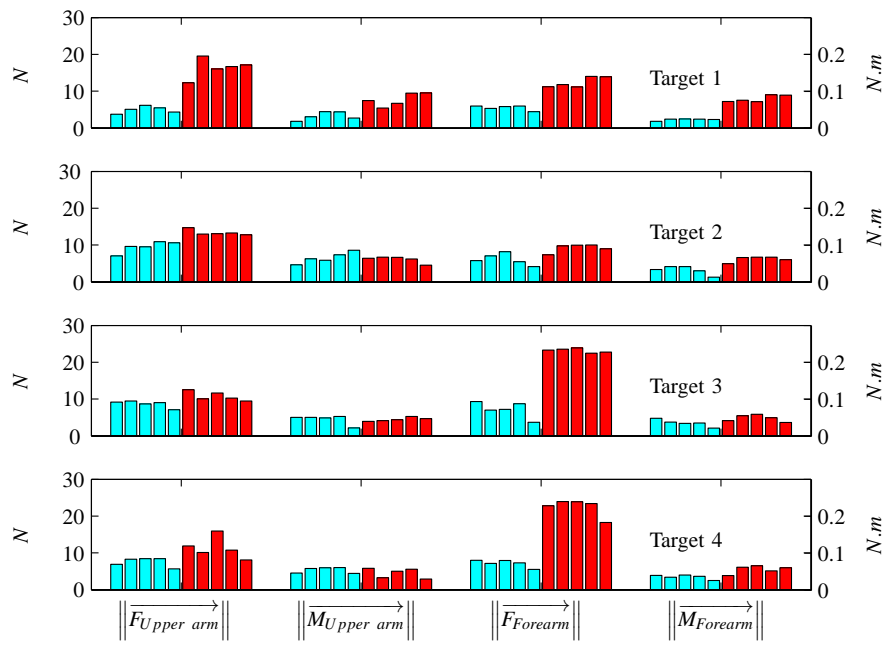

Fig. 20. Incompatible force and moment norm on the fixations averaged of one single subject for each trial to the 4 different targets. The 5 trials with passive DoF fixations are in blue and with classical fixation in red. Trials are chronologically classified, from left to right.

Figure 21 represents the mean across the ten subjects, and the time-averaged force and moment norms.
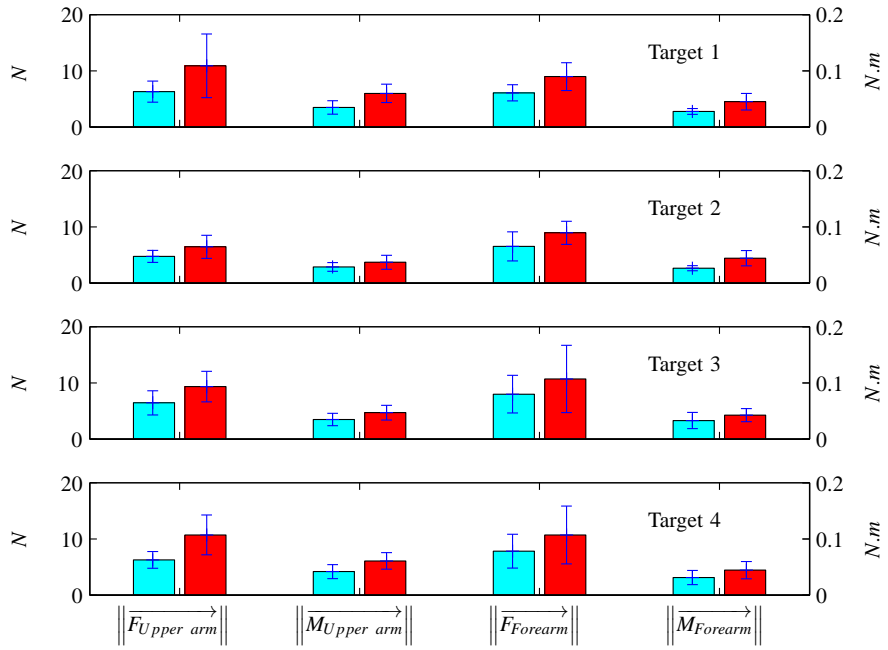

Fig. 21. Incompatible force and moment averaged norm on the fixations averaged over the $10 \operatorname{subjects}\left(F_{x}\right.$ and the three moments $\left.M_{x}, M_{y}, M_{z}\right)$. In red with classical fixation; in blue with passive DoF fixations.

Interestingly, it can be noticed that the standard deviation is lower for the experiments with freed fixations as compared to experiments with locked fixations. We also noticed that, when the subject's forearm length (roughly estimated humerus length) strongly differs from the robot humerus length $(357 \mathrm{~mm})$, then the forces tend to be large during experiments with locked fixations. This is logical from an engineering point of view, since, for hyperstatic systems, the level of force depend on stiffnesses and displacements: when the differences are large between the two kinematic chains, mismatches are larger and the forces that result from these misalignments through the tissue stiffness are larger as well. Meanwhile, for experiments with freed fixations, the amount of measured force did not seem to depend on the subject's kinematic parameters. Again, the fact that the system is not hyperstatic anymore explains this observation. An experimental campaign with more subjects and selected morphologies would still be necessary to obtain statistically consistent results on the influence of the subject's humerus length on the level of forces observed in both conditions. Table II reports the decreases in the level of the incompatible interaction forces.

\begin{tabular}{|c|c|c|c|c|}
\hline Decrease $\%$ & $F_{U \text { pper-arm }}$ & $M_{U \text { pper-arm }}$ & $F_{\text {Forearm }}$ & $M_{\text {Forearm }}$ \\
\hline Target 1 & $42 \%$ & $41 \%$ & $32 \%$ & $38 \%$ \\
\hline Target 2 & $26 \%$ & $22 \%$ & $27 \%$ & $40 \%$ \\
\hline Target 3 & $28 \%$ & $27 \%$ & $22 \%$ & $21 \%$ \\
\hline Target 4 & $41 \%$ & $31 \%$ & $26 \%$ & $29 \%$ \\
\hline
\end{tabular}

TABLE II

DECREASE IN THE LEVEL OF EVERY INCOMPATIBLE COMPONENTS WHEN PASSIVE DOF FIXATIONS ARE USED

In order to statistically evaluate the difference between the two conditions, repeated measures ANOVA were carried out for the force decrease with condition (with passive DoF fixations / without) and target (4 targets) as independent factors. When significant effects were found, a Newman-Keuls post hoc test was applied in order to evaluate the effect of condition on each 
target. The results on the ANOVA obtained are presented both in terms of value of the probability distribution function $\mathrm{F}$, and p-value.

In comparison with previous results obtained in the passive mode experimentation with the manikin, the percentage of decrease of the incompatible force component level is lower, especially for the upper-arm fixation but still statistically
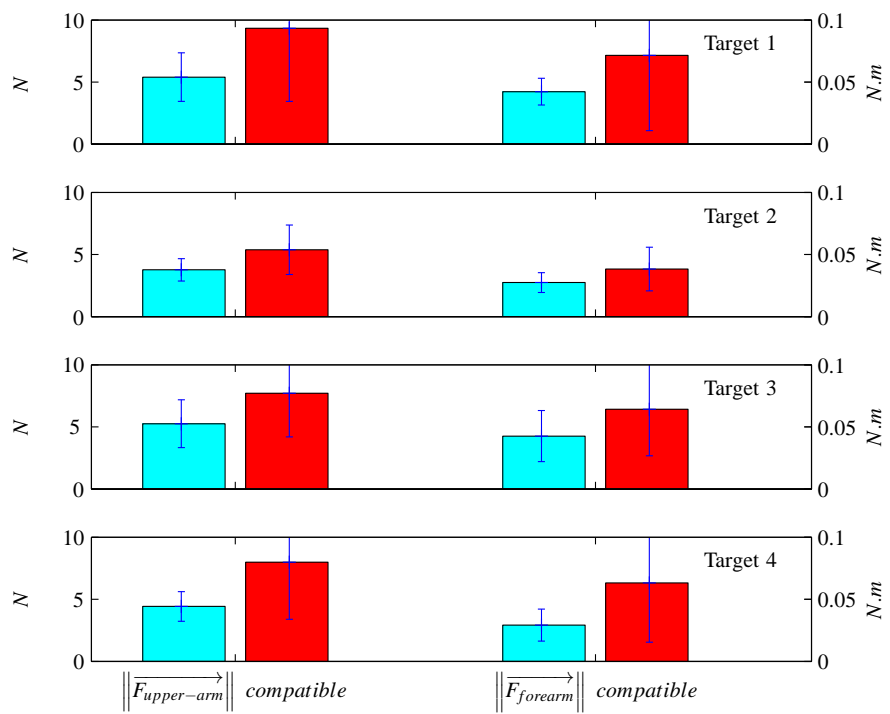

Fig. 22. Compatible force averaged norm on the fixations averaged over the $10 \operatorname{subjects}\left(F_{y}\right.$ and $\left.F_{z}\right)$. In red with classical fixation; in blue with passive DoF fixations.

significant $(\mathrm{F}(1,10)=28.16, p<0.01)$.

This can be explained by the fact that the human limb is much more flexible than the manikin limb. Therefore, hyperstaticity induces lower forces. Interestingly, the force compatible with the passive fixations is also reduced as shown in Fig. 22 $(\mathrm{F}(1,10)=19.46, p<0.01)$.

No statistical significant effects of the nature of the target were found in such results. Nevertheless, several explanations can be formulated to explain the system performance limitations in active mode:

- a bad alignment between the center of rotations of the human joints and the fixations ball joint centers enhanced by the deformations of some parts of the fixation mechanisms,

- use by the subject of its upper limb redundant DoF that are not directly controlled by the robot (wrist and scapula movements), that can completely modify the kinematic sequence.

These hypotheses will be verified in future experimental campaigns.

\section{CONClusion}

In this paper we presented a method aimed at designing the kinematics of fixations between an exoskeleton and a human member. A major result of the theoretical study lies in Equations (7) and (8) that provide the minimal and maximal mobility to be added to each chain, recursively, and lead, by summing up all the components, to Equation (9). Thanks to this method, we built isostatic fixations for a 4-DoF exoskeleton and experimentally verified their benefit on minimizing uncontrollable hyperstatic forces at the human robot interface and thus on a fine control of the interaction forces. These results show that the provided solution effectively limits the level of uncontrolled forces generated by hyperstaticity even in the case of large variations of the human limb geometry, and without requiring a complex adaptable robot structure. Further work could focus on the study of the motion of the passive mechanisms during movements, whish is an indicator on how different are the human motion and robot motion.

\section{ACKNOWLEDGMENTS}

This work was supported in part by the A.N.R. (Agence Nationale de la Recherche) with the project BRAHMA (BioRobotics for Assisting Human Manipulation) PSIROB 2006. Many thanks to Agnès Roby-Brami, Johanna Robertson and Michele Tagliabue for their help in performing experiments with human subjects.

\section{APPENDIX}

\section{A. Demonstration of Proposition 1}

1) Conditions (3) are sufficient: $[(3) \Rightarrow(2)]$.

We here assume that conditions (3) are verified.

Because in $\mathbf{S}_{n}, \mathscr{R}_{i-1}$ is connected on the one side to $\mathscr{R}_{0}$ through $\mathbf{S}_{i-1}$ and on the other side to $\mathscr{R}_{i}$ through $\mathbf{R}_{i}$ (see Fig. 3), one has:

$$
\forall i \in\{1 \ldots n\}, \quad{ }^{\mathbf{S}_{n}} T_{i-1}={ }^{\mathbf{S}_{i-1}} T_{i-1} \cap\left[T_{\mathbf{R}_{i}}+{ }^{\mathbf{S}_{n}} T_{i}\right],
$$

which is a recursive relationship for ${ }^{S_{n}} T_{i}$. Recalling that, by assumption, ${ }^{\mathbf{S}_{n}} \boldsymbol{T}_{\mathbf{S}_{n}}=\{0\}$ (condition $3 \mathrm{c}$ ) and $T_{\mathbf{S}_{i-1}} \cap T_{\mathbf{R}_{i}}=\{0\}$ (condition $3 b$ ), this recursive law trivially leads to (2a).

Furthermore, the kinemato-static duality principle applied to the loop $\left(\mathscr{R}_{0} \rightarrow \mathscr{R}_{i-1} \rightarrow \mathscr{R}_{i} \rightarrow \mathscr{R}_{0}\right)$ in Fig. 3 writes:

$$
\forall i \in\{1 \ldots n\}, \quad \operatorname{dim}\left(\mathbf{S}_{i} W_{\mathbf{L}_{i} \rightarrow 0}\right)+\operatorname{dim}\left(T_{\mathbf{S}_{i-1}}+T_{\mathbf{R}_{i}}+T_{\mathbf{L}_{i}}\right)=6 .
$$

Thanks to condition (3a), this leads to:

$$
\forall i \in\{1 \ldots n\}, \quad \mathbf{S}_{i} W_{\mathbf{L}_{i} \rightarrow 0}=\{0\} .
$$

Considering again the system $\mathbf{S}_{i}$ depicted in Fig. 3, and recalling that $\mathbf{L}_{i}$ and $\mathbf{R}_{i}$ are serial chains, one has, $\forall i \in\{1 \ldots n\}$ :

$$
{ }^{\mathbf{S}_{i}} W_{\mathbf{L}_{i} \rightarrow 0}={ }^{\mathbf{S}_{i}} W_{\mathbf{L}_{i} \rightarrow i}={ }^{\mathbf{s}_{i}} W_{\mathbf{R}_{i} \rightarrow i}={ }^{\mathbf{S}_{i}} W_{\mathbf{R}_{i} \rightarrow i-1}=\{0\} .
$$

Therefore, statically speaking, the multi-loop system $\mathbf{S}_{i-1}$ is in the same state when included in $\mathbf{S}_{i}$ than when isolated from the rest of the mechanism.

$$
\forall i \in\{2 \ldots n\}, \quad{ }^{\mathbf{S}_{i}} W_{\mathbf{L}_{i-1} \rightarrow 0}={ }^{\mathbf{S}_{i-1}} W_{\mathbf{L}_{i-1} \rightarrow 0},
$$

which, together with (13) recursively leads to condition (2b).

2) Conditions (3) are necessary : $[\overline{(3)} \Rightarrow \overline{(2)}]$.

Firstly, if condition (3c) is not verified, then $\mathbf{S}_{n} T_{n}=T_{\mathbf{S}_{n}} \neq\{0\}$. In this case, (2a) is not satisfied.

Secondly, if (3b) is not verified, then $\exists i,\left(T_{\mathbf{R}_{i}} \cap T_{\mathbf{S}_{i-1}}\right) \neq\{0\}$. Thanks to Equation (11), this leads to:

$$
\exists i \in\{1 \cdots n\}, \quad{ }^{\mathbf{S}_{n}} T_{i-1} \neq\{0\},
$$


which directly contradicts (2a).

Thirdly, if (3a) is not verified, i.e.:

$$
\exists i, \operatorname{dim}\left(T_{\mathbf{S}_{i-1}}+T_{\mathbf{R}_{i}}+T_{\mathbf{L}_{i}}\right) \leq 6,
$$

then $\exists i, \quad \mathbf{S}_{i} W_{\mathbf{L}_{i \rightarrow 0}} \neq\{0\}$, meaning that $\mathbf{S}_{i}$ taken isolate is hyperstatic. Obviously, adding the rest of the mechanism to build $\mathbf{S}_{n}$, which consists of adding a parallel branch to $\mathbf{S}_{i}$ between $\mathscr{R}_{0}$ and $\mathscr{R}_{i}$ will not decrease the degree of hyperstaticity. Therefore $\exists i, \mathbf{S}_{n} W_{\mathbf{L}_{i \rightarrow 0}} \neq\{0\}$, which contradicts condition $(2 b)$.

B. Singularity analysis for ABLE and the two proposed fixation mechanisms

Let us take the mechanism depicted in Figure 23: $\mathbf{R}_{1}$ is a ball joint which center is $M_{1} ; \mathbf{L}_{1}$ is composed of a ball joint whose center is $P_{1}$ (with $\overrightarrow{M_{1} P_{1}}=l_{1} \cdot \overrightarrow{z_{1}}$ and $l_{1} \neq 0$ ) and a slide along $\left(P_{1}, \overrightarrow{z_{a r m}}\right) ; \mathbf{R}_{2}$ is a pivot joint whose axis is $\left(M_{2}, \overrightarrow{x_{2}}\right) ; \mathbf{L}_{2}$ is composed of a ball joint whose center is $P_{2}$ (with $\overrightarrow{M_{2} P_{2}}=l_{2} \cdot \overrightarrow{z_{a}}$ and $\left.l_{2} \neq 0\right)$ and a slide along $\left(P_{2}, \overrightarrow{z_{f}}\right)$.

In order to find the singular configurations of this system, we use the necessary and sufficient conditions (3).

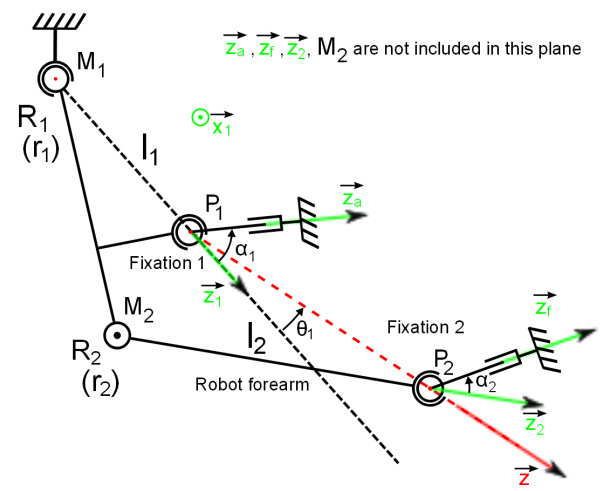

Fig. 23. Kinematics of ABLE + its fixations. The plane of the figure, perpendicular to $\vec{x}_{1}$, is defined by $M_{1}, P_{1}$ and $P_{2}$ while $M_{2}$ is outside the plane.

\section{1) Examination of Condition (3a)}

- For $i=1$, (3a) writes $\operatorname{dim}\left(T_{\mathbf{R}_{1}}+T_{\mathbf{L}_{1}}\right)=6$.

At point $P_{1}$, velocities allowed by $\mathbf{L}_{1}$ belong to the vector subspace $T_{\mathbf{L}_{1}}=\operatorname{span}\left\{t_{1}, t_{2}, t_{3}, t_{4}\right\}$ and the velocities allowed by $\mathbf{R}_{1}$ belong to $T_{\mathbf{R}_{1}}=\operatorname{span}\left\{t_{5}, t_{6}, t_{3}\right\}$, with

$$
\begin{gathered}
t_{1}=\left(x_{1}{ }^{\mathrm{T}} 0_{3}{ }^{\mathrm{T}}\right)^{\mathrm{T}}, \quad t_{3}=\left(z_{1}{ }^{\mathrm{T}} 0_{3}{ }^{\mathrm{T}}\right)^{\mathrm{T}}, \quad t_{5}=\left(x_{1}{ }^{\mathrm{T}}-l_{1} \cdot y_{1}{ }^{\mathrm{T}}\right)^{\mathrm{T}} \\
t_{2}=\left(y_{1}{ }^{\mathrm{T}} 0_{3}{ }^{\mathrm{T}}\right)^{\mathrm{T}}, \quad t_{4}=\left(0_{3}{ }^{\mathrm{T}} z_{a}{ }^{\mathrm{T}}\right)^{\mathrm{T}}, \quad t_{6}=\left(y_{1}{ }^{\mathrm{T}} l_{1} \cdot x_{1}{ }^{\mathrm{T}}\right)^{\mathrm{T}}
\end{gathered}
$$

Thus $T_{\mathbf{R}_{1}}+T_{\mathbf{L}_{1}}=\operatorname{span}\left\{t_{1}, \ldots, t_{6}\right\}$. Defining

$t_{5}^{\prime}=\frac{\left(t_{6}-t_{2}\right)}{l_{1}}=\left(0_{3}{ }^{\mathrm{T}} x_{1}{ }^{\mathrm{T}}\right)^{\mathrm{T}}$ and $t_{6}^{\prime}=\frac{\left(t_{1}-t_{5}\right)}{l_{1}}=\left(0_{3}{ }^{\mathrm{T}} y_{1}{ }^{\mathrm{T}}\right)^{\mathrm{T}}$, we can easily show that

$$
\left[\begin{array}{llllll}
t_{1} & t_{2} & t_{3} & t_{4} & t_{5}^{\prime} & t_{6}^{\prime}
\end{array}\right]=A\left[\begin{array}{llllll}
t_{1} & t_{2} & t_{3} & t_{4} & t_{5} & t_{6}
\end{array}\right]
$$

with $\operatorname{det}(A)=\frac{1}{l_{1}^{2}}$. Since $l_{1} \neq 0, \tau_{1}=\left\{t_{1}, . ., t_{6}\right\}$ is a basis of $\mathbb{R}^{6}$ if and only if $\tau_{2}=\left\{t_{1}, . ., t_{4}, t_{5}^{\prime}, t_{6}^{\prime}\right\}$ is a basis of $\mathbb{R}^{6}$. Let us consider now $a_{i} \in \mathbb{R}, i \in\{1, \ldots, 6\}$ such that:

$$
a_{1} t_{1}+a_{2} t_{2}+a_{3} t_{3}+a_{4} t_{4}+a_{5} t_{5}^{\prime}+a_{6} t_{6}^{\prime}=0
$$

This equation is equivalent to :

$$
\left\{\begin{array}{c}
a_{1} \overrightarrow{x_{1}}+a_{2} \overrightarrow{y_{1}}+a_{3} \overrightarrow{z_{1}}=\overrightarrow{0} \\
a_{4} \overrightarrow{z_{a}}+a_{5} \overrightarrow{x_{1}}+a_{6} \overrightarrow{y_{1}}=\overrightarrow{0}
\end{array}\right.
$$

Since $\left(\overrightarrow{x_{1}}, \overrightarrow{y_{1}}, \overrightarrow{z_{1}}\right)$ is a basis, (18) is equivalent to

$$
\left\{\begin{array}{l}
a_{1}=a_{2}=a_{3}=0 \\
a_{4} d_{z}=0 ; \quad a_{6}+a_{4} d_{y}=0 ; \quad a_{5}+a_{6} d_{x}=0 ;
\end{array}\right.
$$

where $\overrightarrow{z_{\text {bras }}}=d_{x} \overrightarrow{x_{1}}+d_{y} \overrightarrow{y_{1}}+d_{z} \overrightarrow{z_{1}}$. If $d_{z} \neq 0$ then (19) implies $\forall i \in\{1 \cdots 6\} a_{i}=0$ and the $\tau_{2}$ et $\tau_{1}$ family are basis of $\mathbb{R}^{6}$.

Otherwise, there exists a non null combination of $a_{i}$ that verifies (17). Condition (3a) is thus verified for $i=1$ if and only if $\overrightarrow{z_{a}} \cdot \overrightarrow{z_{1}} \neq 0$. This is a singular value to be avoided. In the rest of the study we will thus consider that $\overrightarrow{z_{a}} \cdot \overrightarrow{z_{1}} \neq 0$.

- For $i=2$, (3a) writes $\operatorname{dim}\left(T_{\mathbf{S}_{1}}+T_{\mathbf{R}_{2}}+T_{\mathbf{L}_{2}}\right)=6$.

We know that $T_{\mathbf{S}_{1}}=T_{\mathbf{R}_{1}} \cap T_{\mathbf{L}_{1}}$. Let us consider $t \in T_{\mathbf{L}_{1}}$ and $t^{\prime} \in T_{\mathbf{R}_{1}}$. One has:

$$
\begin{aligned}
\exists\left(\alpha_{1}, \alpha_{2}, \alpha_{3}, \alpha_{4}\right) \text { such that } t & =\sum_{i=1}^{4} \alpha_{i} t_{i} \\
\exists\left(\alpha_{1}^{\prime}, \alpha_{2}^{\prime}, \alpha_{3}^{\prime},\right) \quad \text { such that } t^{\prime} & =\alpha_{1}^{\prime} t_{5}+\alpha_{2}^{\prime} t_{6}+\alpha_{3}^{\prime} t
\end{aligned}
$$

Using $\overrightarrow{z_{a}} \cdot \overrightarrow{z_{1}} \neq 0$, one easily gets:

$$
t=t^{\prime} \Leftrightarrow \alpha_{1}=\alpha_{2}=\alpha_{4}=\alpha_{1}^{\prime}=\alpha_{2}^{\prime}=0 .
$$

or:

$$
t=t^{\prime} \Leftrightarrow t=\alpha_{3} t_{3}=\alpha_{3}^{\prime} t_{3} .
$$

In other words, at point $P_{1}$ :

$$
T_{\mathbf{S}_{1}}=T_{\mathbf{R}_{1}} \cap T_{\mathbf{L}_{1}}=\operatorname{span}\left(\left\{t_{3}\right\}\right)=\operatorname{span}\left(\left\{\left(z_{1}{ }^{\mathrm{T}} 0_{3}{ }^{\mathrm{T}}\right)^{\mathrm{T}}\right\}\right) .
$$

We know write twists at point $P_{2}$. We get: $T_{\mathbf{S}_{1}}=\operatorname{span}\left(\left\{t_{7}\right\}\right)$, $T_{\mathbf{R}_{2}}=\operatorname{span}\left(\left\{t_{8}\right\}\right)$ and $T_{\mathbf{L}_{2}}=\operatorname{span}\left(\left\{\begin{array}{llll}t_{9} & t_{10} & t_{11} & t_{12}\end{array}\right\}\right)$, with:

$t_{7}=\left(z_{1}{ }^{\mathrm{T}} l \sin \theta_{1} x_{1}{ }^{\mathrm{T}}\right)^{\mathrm{T}}, \quad t_{8}=\left(x_{2}{ }^{\mathrm{T}}-l_{2} y_{2}{ }^{\mathrm{T}}\right)^{\mathrm{T}}, \quad t_{9}=\left(x_{2}{ }^{\mathrm{T}} 0^{\mathrm{T}}\right)^{\mathrm{T}}$

$$
t_{10}=\left(y_{2}{ }^{\mathrm{T}} 0^{\mathrm{T}}\right)^{\mathrm{T}}, \quad t_{11}=\left(z_{2}{ }^{\mathrm{T}} 0^{\mathrm{T}}\right)^{\mathrm{T}}, \quad t_{12}=\left(0^{\mathrm{T}} z_{f}^{\mathrm{T}}\right)^{\mathrm{T}},
$$

where $\overrightarrow{P_{1} P_{2}}=: l \vec{z}$ and $\theta_{1}:=\left(\overrightarrow{\overrightarrow{z_{1}}, \vec{z}}\right)$ measured around $\vec{x}_{1}$. Thus $T_{\mathbf{S}_{1}}+T_{\mathbf{R}_{2}}+T_{\mathbf{L}_{2}}=\operatorname{span}\left(\left\{t_{7}, t_{8}, t_{9}, t_{10}, t_{11}, t_{12}\right\}\right)$.

Suppose first that $\sin \theta_{1}=0$. Then, denoting $\overrightarrow{z_{1}}=z_{1 x} \cdot \overrightarrow{x_{2}}+$ $z_{1 y} \cdot \overrightarrow{y_{2}}+z_{1 z} \cdot \overrightarrow{z_{2}}$, one gets:

$$
t_{7}=z_{1 x} t_{9}+z_{1 y} t_{10}+z_{1 z} t_{12}
$$

In this particular case, $\left\{t_{7} \ldots t_{12}\right\}$ is not a basis, which identifies a second singular configuration, when $M_{1}, P_{1}$ and $P_{2}$ are aligned. In the rest of the study we will thus assume that this singular configuration is also avoided, that is: $\sin \theta_{1} \neq 0$. Defining

$$
\begin{aligned}
t_{7}^{\prime} & =\frac{\left(t_{7}-z_{1 x} t_{9}-z_{1 y} t_{10}-z_{1 z} t_{12}\right)}{l \sin \theta_{1}}=\left(\begin{array}{ll}
0^{\mathrm{T}} & x_{1}{ }^{\mathrm{T}}
\end{array}\right)^{\mathrm{T}}, \text { and } \\
t_{8}^{\prime} & =\frac{\left(t_{10}-t_{8}\right)}{l_{2}}=\left(0^{\mathrm{T}} y_{2}{ }^{\mathrm{T}}\right)^{\mathrm{T}},
\end{aligned}
$$

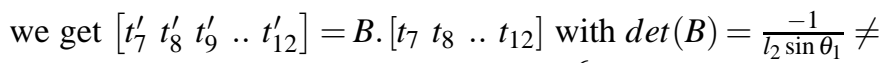
0 . Thus $\tau_{3}=\left\{t_{7} \ldots t_{12}\right\}$ is a basis of $\mathbb{R}^{6}$ if and only if $\tau_{4}=$ $\left\{t_{7}^{\prime} \ldots t_{12}^{\prime}\right\}$ is a basis of $\mathbb{R}^{6}$. Let us consider $b_{i} \in \mathbb{R}, i \in\{1, . ., 6\}$ such that:

$$
b_{1} t_{7}^{\prime}+b_{2} t_{8}^{\prime}+b_{3} t_{9}+b_{4} t_{10}+b_{5} t_{11}+b_{6} t_{12}=0 .
$$


It comes easily that $b_{3}=b_{4}=b_{5}=0$ and $b_{1} t_{7}^{\prime}+b_{2} t_{8}^{\prime}+b_{6} t_{12}^{\prime}=0$ which is equivalent to $b_{1} \overrightarrow{x_{1}}+b_{2} \overrightarrow{y_{2}}+b_{6} \overrightarrow{z_{f}}=\overrightarrow{0}$. The necessary and sufficient conditions to have a non-null triplet $b_{1}, b_{2}, b_{6}$ verifying the previous equation is that $\overrightarrow{x_{1}}, \overrightarrow{y_{2}}, \overrightarrow{z_{f}}$ are coplanar. This identifies a third singularity, which, again, is supposed to be avoided in the rest of the study.

2) Examination of the condition (3b)

- For $i=1$, since $T_{\mathbf{S}_{0}}=\{0\}$, one directly gets $\operatorname{dim}\left(T_{\mathbf{S}_{0}} \cap\right.$ $\left.T_{\mathbf{L}_{1}}\right)=0$.

- For $i=2$, it is necessary to verify that $\operatorname{dim}\left(T_{\mathbf{S}_{1}} \cap T_{\mathbf{L}_{2}}\right)=0$.

Let us consider $t \in T_{\mathbf{S}_{1}}$ and $t^{\prime} \in T_{\mathbf{L}_{2}}$. One has:

$$
\begin{aligned}
\exists \alpha_{1} & \in \mathbb{R} \quad / \quad t=\alpha_{1} t_{7} \\
\exists \alpha_{1}^{\prime}, \alpha_{2}^{\prime}, \alpha_{3}^{\prime}, \alpha_{4}^{\prime} & \in \mathbb{R} \quad / \quad t^{\prime}=\alpha_{1}^{\prime} t_{9}+\alpha_{2}^{\prime} t_{10}+\alpha_{3}^{\prime} t_{11}+\alpha_{4}^{\prime} t_{12} .
\end{aligned}
$$

One easily shows that $t=t^{\prime}$ is equivalent to:

$$
\left\{\begin{array}{l}
\alpha_{1} l \sin \theta_{1} \overrightarrow{x_{1}}+\alpha_{4}^{\prime} \overrightarrow{z_{f}}=\overrightarrow{0} \\
\left(\alpha_{1} z_{1 x}+\alpha_{1}^{\prime}\right) \overrightarrow{x_{2}}+\left(\alpha_{1} z_{1 y}+\alpha_{2}^{\prime}\right) \overrightarrow{y_{2}}+\left(\alpha_{1} z_{1 z}+\alpha_{3}^{\prime}\right) \overrightarrow{z_{2}}=\overrightarrow{0}
\end{array}\right.
$$

Since $\overrightarrow{x_{1}}$ is not colinear to $\overrightarrow{z_{f}}$, the first equation leads to $\alpha_{1}=$ $\alpha_{4}^{\prime}=0$. Similarly, since $\left\{\overrightarrow{x_{2}}, \overrightarrow{y_{2}}, \overrightarrow{z_{2}}\right\}$ forms a basis, $\alpha_{1}^{\prime}=\alpha_{2}^{\prime}=$ $\alpha_{3}^{\prime}=0$. In conclusion, $\operatorname{dim}\left(T_{\mathbf{S}_{1}} \cap T_{\mathbf{L}_{2}}\right)=\{0\}$.

3) Examination of the condition (3c)

For the considered example, $n=2$ and condition (3c) writes $\operatorname{dim}\left(T_{\mathbf{S}_{2}}\right)=0$. Since $T_{\mathbf{S}_{2}}=\left(T_{\mathbf{S}_{1}}+T_{\mathbf{R}_{2}}\right) \cap T_{\mathbf{L}_{2}}$, we need to verify that any vector that belongs to both $\left(T_{\mathbf{S}_{1}}+T_{\mathbf{R}_{2}}\right)$ and $T_{\mathbf{L}_{2}}$ is null. Let us consider $t \in\left(T_{\mathbf{S}_{1}}+T_{\mathbf{R}_{2}}\right)$ and $t^{\prime} \in T_{\mathbf{L}_{2}}$. One has:

$$
\begin{aligned}
\exists \alpha_{1}, \alpha_{2} \in \mathbb{R} \quad / \quad t=\alpha_{1} t_{7}+\alpha_{2} t_{8} \\
\exists \alpha_{1}^{\prime}, . ., \alpha_{4}^{\prime} \in \mathbb{R} \quad / \quad t^{\prime}=\alpha_{1}^{\prime} t_{9}+\alpha_{2}^{\prime} t_{1} 0+\alpha_{3}^{\prime} t_{1} 1+\alpha_{4}^{\prime} t_{1} 2
\end{aligned}
$$

Therefore $t=t^{\prime}$ is equivalent to:

$$
\left\{\begin{array}{l}
\alpha_{1} l \sin \theta_{1} \overrightarrow{x_{1}}-\alpha_{2} l_{2} \overrightarrow{y_{2}}+\alpha_{4}^{\prime} \overrightarrow{z_{f}}=\overrightarrow{0} \\
\left(\alpha_{1} z_{1 x}+\alpha_{1}^{\prime}+\alpha_{2}\right) \overrightarrow{x_{2}}+\left(\alpha_{1} z_{1 y}+\alpha_{2}^{\prime}\right) \overrightarrow{y_{2}}+\left(\alpha_{1} z_{1 z}+\alpha_{3}^{\prime}\right) \overrightarrow{z_{2}}=\overrightarrow{0}
\end{array}\right.
$$

The first of these two equations leads to $\alpha_{1}=\alpha_{2}=\alpha_{4}^{\prime}=0$ since it is supposed that $\overrightarrow{x_{1}}, \overrightarrow{y_{2}}$ and $\overrightarrow{z_{f}}$ are not coplanar in order to avoid the third singularity, and $\sin \theta_{1} \neq 0$ in order to avoid the second singularity. Therefore, the second equation leads to $\alpha_{1}=\alpha_{2}=\alpha_{4}^{\prime}=0$ because $\left\{\overrightarrow{x_{2}}, \overrightarrow{y_{2}}, \overrightarrow{z_{2}}\right\}$ forms a basis. In conclusion, $t=t^{\prime} \Rightarrow t=0$, thus $\operatorname{dim}\left(T_{\mathbf{S}_{2}}\right)=0$.

4) Summary.

In conclusion, we identified three singularities:

1) $\overrightarrow{z_{a}} \cdot \overrightarrow{z_{1}}=0$ representing the case where the passive slide, mounted parallel to the upper arm axis, is perpendicular to the robot upper limb axis. This case will never appear in practice since the angle between $\overrightarrow{z_{a}}$ and $\overrightarrow{z_{1}}$ reflects small discrepancies between the exoskeleton and human kinematics, and remains smaller than a few degrees.

2) $\sin \left(\theta_{1}\right)=0$ representing the case where $M_{1}, P_{1}$ and $P_{2}$ are aligned. This singular configuration can be avoided by limiting the range of motion for the robot elbow to a few degrees before full extension. Note that the full extension of the human arm is the same singularity and thus it cannot be avoided.

3) $\overrightarrow{x_{1}}, \overrightarrow{y_{2}}$ and $\overrightarrow{z_{f}}$ coplanar. This configuration does not appear in practice, since in the nominal configuration, $\overrightarrow{x_{1}}$ is perpendicular to the plane generated by $\overrightarrow{y_{2}}$ and $\overrightarrow{z f}$.

Therefore, under normal conditions of operation, the ABLE exoskeleton with its two fixations never falls into a singular configuration.

\section{REFERENCES}

[1] N. Jarrasse and G. Morel. A formal method for avoiding hyperstaticity when connecting an exoskeleton to a human member. In Robotics and Automation (ICRA), 2010 IEEE International Conference on, pages 1188 -1195 , May 2010.

[2] N. Jarrasse and G. Morel. On the kinematic design of exoskeletons and their fixations with a human member. In Proceedings of Robotics: Science and Systems (RSS'2010), June 2010.

[3] A.B. Zoss, H. Kazerooni, and A. Chu. Biomechanical design of the berkeley lower extremity exoskeleton (bleex). Mechatronics, IEEE/ASME Transactions on, 11(2):128 -138, april 2006.

[4] M. Mihelj, T. Nef, and R. Riener. Armin ii - 7 dof rehabilitation robot: mechanics and kinematics. In Robotics and Automation, 2007 IEEE International Conference on, pages 4120 -4125, 10-14 2007.

[5] A. Frisoli, L. Borelli, A. Montagner, S. Marcheschi, C. Procopio, F. Salsedo, M. Bergamasco, M.C. Carboncini, M. Tolaini, and B. Rossi. Arm rehabilitation with a robotic exoskeleleton in virtual reality. In Rehabilitation Robotics, 2007. ICORR 2007. IEEE 10th International Conference on, pages $631-642$, june 2007.

[6] J.C. Perry, J. Rosen, and S. Burns. Upper-limb powered exoskeleton design. Mechatronics, IEEE/ASME Transactions on, 12(4):408 -417, aug. 2007.

[7] Jose L. Pons. Wearable Robots: Biomechatronic Exoskeletons. Wiley, April 2008.

[8] Stephen H. Scott and David A. Winter. Biomechanical model of the human foot: Kinematics and kinetics during the stance phase of walking. Journal of Biomechanics, 26(9):1091-1104, September 1993.

[9] Leboucher J., Lempereur M., Burdin V., and Remy-Neris O. Radius movement simulation based on articular surfaces. In Computational Systems bioinformatics conference, 2010.

[10] F.C.T. Van der Helm, H.E.J. Veeger, G.M. Pronk, L.H.V. Van der Woude, and R.H. Rozendal. Geometry parameters for musculoskeletal modelling of the shoulder system. Journal of Biomechanics, 25(2):129144, February 1992.

[11] A. Schiele. An explicit model to predict and interpret constraint force creation in phri with exoskeletons. In Robotics and Automation, 2008. ICRA 2008. IEEE International Conference on, pages $1324-1330,19-$ 232008.

[12] A. Schiele and F.C.T. van der Helm. Kinematic design to improve ergonomics in human machine interaction. Neural Systems and Rehabilitation Engineering, IEEE Transactions on, 14(4):456 -469, dec. 2006.

[13] S.J. Housman, L. Vu, T. Rahman, R.J. Sanchez, and D.J. Reinkensmeyer. Arm-Training with T-WREX after chronic stroke: Preliminary results of a randomized controlled trial. In Rehabilitation Robotics, 2007. ICORR 2007. IEEE 10th International Conference on, pages 562-568, June 2007.

[14] L W Lamoreux. Kinematic measurements in the study of human walking. Bull Prosthet Res., 10(15):3-84, 1971. PMID: 5131748.

[15] KL Markolf, JS Mensch, and HC Amstutz. Stiffness and laxity of the knee-the contributions of the supporting structures. a quantitative in vitro study. J Bone Joint Surg Am, 58(5):583-594, 1976.

[16] C. Diez-Martnez, J. Rico, J. Cervantes-Snchez, and J. Gallardo. Mobility and connectivity in multiloop linkages. In Advances in Robot Kinematics, pages 455-464. 2006.

[17] M. Stokdijk, C. G. M. Meskers, H. E. J. Veeger, Y. A. de Boer, and P. M. Rozing. Determination of the optimal elbow axis for evaluation of placement of prostheses. Clinical Biomechanics, 14(3):177-184, March 1999.

[18] Grigore Gogu. Mobility and spatiality of parallel robots revisited via theory of linear transformations. European Journal of Mechanics A/Solids, 24(4):690 - 711, 2005.

[19] J. M. Rico, J. Gallardo, and B. Ravani. Lie algebra and the mobility of kinematic chains. Journal of Robotic Systems, 20(8):477-499, 2003.

[20] K. J. Waldron. The constraint analysis of mechanisms. Journal of Mechanisms, 1(2):101-114, 1966. 
[21] Garrec. P. French patent: Transmission vis, ecrou et cable attache a la vis - fr0101630, 2000 (eur 01938347.0-2421 and us 10/296,740 (screw and nut transmission and cable). 2000.

[22] P. Garrec, J.P. Friconneau, Y. Measson, and Y. Perrot. Able, an innovative transparent exoskeleton for the upper-limb. Intelligent Robots and Systems, 2008. IROS 2008. IEEE/RSJ International Conference on, pages 1483-1488, Sept. 2008

[23] N. Jarrasse, M. Tagliabue, J.V.G. Robertson, A. Maiza, V. Crocher, A. Roby-Brami, and G. Morel. A methodology to quantify alterations in human upper limb movement during co-manipulation with an exoskeleton. Neural Systems and Rehabilitation Engineering, IEEE Transactions on, 18(4):389 -397, 2010.

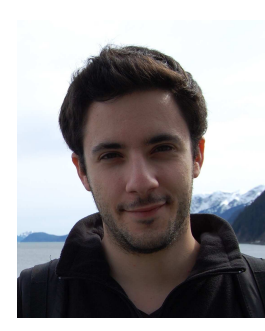

Nathanael Jarrassé received his degree in Industrial Systems Engineering from the Ecole Nationale Supérieure d'Arts et Métiers (ENSAM) and a Master of Science degree in Mechanics and System Engineering in 2006 along with a $\mathrm{PhD}$ in robotics (2010), in Univ. P\&M Curie, France. He is now a researcher at CNRS-ISIR and currently visiting researcher at the Department of Bioengineering at the Imperial College London. His work focuses on rehabilitation robotics, kinetostatic analysis, physical ment analysis and transparency. Human-Robot interaction, interaction control, move-

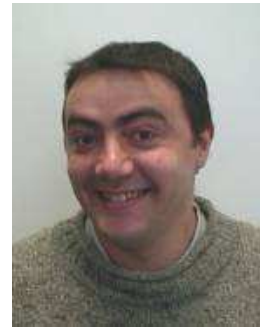

Guillaume Morel received a M.S. in electrical engineering (1990) and a $\mathrm{PhD}$ in mechanical engineering (1994), in Univ. P\&M Curie, France. After a postdoc at M.I.T. and a first assistant professorship in Strabsourg, he came back to Paris in 2001. Over these years, his reserach interests have been force feedback control and visual servoing of robots, with, for the last decade, a particular focus on medical applications. He now heads a multlidisciplinary group developing devices aimed at assisting gesture through the concept of comanipulation. 